\title{
Managing sex offenders: is there a role for psychiatry?
}

The issue of child sexual abuse (CSA) has become increasingly impossible for Irish society to ignore, as decades of denial and disbelief give way before the torrent of disclosures of abuse that has occurred over many decades in institutions, family homes, schools and so on. Even so, these disclosures represent only the tip of the iceberg that abuse represents, as most instances of abuse are never reported to any authority, or even to friends or family. The recently published report Sexual Abuse and Violence in Ireland' suggests that over $20 \%$ of girls and $16 \%$ of boys experience contact sexual abuse before the age of 17 years. Approximately half of these victims had never disclosed the abuse prior to participation in the study. The social, psychological and economic costs of this abuse remain to be properly investigated, but are likely to be enormous, with estimates of the compensation awards to victims of institutional abuse alone running to at least several hundred million euro. The trail of individual emotional distress and disrupted families left in the wake of this abuse is only beginning to emerge. Thus, the importance of CSA as an urgent social issue has never been clearer.

\section{Psychiatric illness in sex offenders}

In order to prevent further CSA, the early identification, assessment and treatment of the perpetrators of sexual abuse is of fundamental importance. Severe mental illness may be an aetiological factor in a small minority of cases of sexual assault. The prevalence of psychotic illness among sex offenders has been reported as in the range $5 \%-8 \%,{ }^{2}$ which is significantly higher than the general population though comparable to the prevalence rate for non-sex offenders. A small number of studies indicate that in men who suffer from major psychiatric illness (especially psychosis), the mental illness appears to play an important or primary role in their sexual aggression. ${ }^{3}$ In those cases where the sexual offence has been driven by psychotic illness, psychiatric treatment including pharmacotherapy will be the primary treatment modality.

Apart from psychotic disorders, sex offenders have been found to suffer from a very high prevalence of other Axis 1 psychiatric disorders. A recent study of men with a paraphilia or paraphilia-related disorder has reported prevalence rates for major depression, anxiety disorders and substance abuse disorders of approximately $40 \%$ in each case, while retrospectively diagnosed ADHD was found to have a prevalence of over $35 \%$ in paraphilia. ${ }^{4}$ In a sample of child molesters, the

Pat Gibbons, MD, Consultant Psychiatrist, Kildare and West Wicklow Psychiatric Service, Ireland.

SUBMITTED: FEBRUARY 19, 2003. ACCEPTED: FEBRUARY 28, 2003 lifetime prevalence of mood disorders was found to be $67 \%$, with $60 \%$ being diagnosed with a substance misuse disorder and a remarkable $93 \%$ of subjects meeting the criteria for any DSM IV Axis 1 disorder. $^{5}$

Alcohol intoxication appears to be a particular risk factor for sexual aggression in rapists, ${ }^{6}$ while altered mood states, ie. anxiety or depression, constitute a similar risk factor in child molesters. ${ }^{2}$ Clearly, apart from the obligation to provide humane and comprehensive treatment for whatever psychiatric disorder that an offender might suffer from, such treatment is a prerequisite for successful participation in psychotherapy aimed at the paraphilia itself, and therefore has a potential impact on sexual recidivism. The very high risk of co-morbid mood and other disorders in this population requires psychiatrists to be intimately involved in the assessment and provision of treatment to offenders from an early stage.

Assessment and treatment of sexual aggression

While treatment programmes for sexual aggression per se have proliferated in the $\mathrm{UK}^{7}$ and $\mathrm{US}^{2}$ in recent years, there has been only a minimal increase in the availability of treatment for sex offenders in Ireland, either within the prison service or for offenders on probation in the community. Up to the $1980 \mathrm{~s}$, most treatment programmes for sex offenders were based on either a psychodynamic or a purely behavioural approach (eg. aversive conditioning to decrease deviant sexual arousal and social/sexual skills training to improve appropriate functioning). Since the 1980s, however, treatment has been largely based on a combination of cognitive-behavioural therapy (CBT), psychosexual education and social skills work, relapse prevention and intensive supervision by the probation services in both the UK ${ }^{7}$ and in the US. ${ }^{2}$ Most of these programmes are lead by probation officers, often with support from forensic psychology services. The average length of time in treatment is 81 hours (range 54150 hours). Most of the participants are child molesters, because of a belief that rapists are less motivated to receive treatment and are more difficult to treat. ${ }^{\text {? }}$

The essential focus of therapy is on challenging the distortions in cognitive processing that allow the offender to deny or minimise their responsibility for the sexual offence, or minimise the recognition of harm done to the victim and thereby protect the offender from shame and guilt. ${ }^{8}$ In many cases, prior to discharge, therapy will focus on identifying the events that precede lapses and relapses with the aim of breaking this chain of events (relapse prevention), while social and sexual skills therapy aims to improve the offender's functioning in the context of age-appropriate intimate relationships. Clearly, this multiple modality approach is likely to 
be most effective with coordinated input from a number of professional disciplines.

As increasing emphasis is put of the development of cognitive-behavioural psychotherapy skills as part of basic training, many psychiatrists are equipped with the essential skills required to participate in the psychological treatment of offenders. In addition to the provision of psychotherapy, the psychiatrist can play an important role in coordinating other aspects of treatment that have been shown to have a positive impact in reducing the risk of recidivism, including pharmacotherapy. In terms of the efficacy of the various treatment modalities, the evidence is somewhat ambiguous, with both cognitive behavioural and pharmacological treatments showing positive evidence of efficacy. ${ }^{9}$ Nevertheless, serious reservations have been raised in relation to hormonal treatments not only due to ethical reservations about their use in both voluntary and involuntary or court-mandated contexts ${ }^{10}$ but also because of high drop-out rates due to side-effects. There is some evidence that a combination of both psychological and drug treatments may provide the best treatment outcome. ${ }^{11}$ Whatever the chosen treatment option, it is clear that the psychiatrist can play an important coordinating role in the multidisciplinary treatment of offenders.

\section{Pharmacological treatment of sexual aggression}

Anti-androgenic treatment is the longest established drug treatment for sexual offenders. Cyproterone acetate (CPA) is an anti-androgenic drug which is used in a number of different medical conditions such as prostate cancer but has been used to decrease sex drive, and therefore the risk of sexual reoffending, for over three decades. CPA is felt to work by disrupting androgen receptors, thereby blocking intra-cellular uptake of testosterone ${ }^{12}$ and also decreasing serum testosterone levels. Medroxy-progesterone acetate (MPA) is felt to work by decreasing testosterone production in the testes, by increasing the activity of testosterone reductase activity in the liver and by displacing testosterone from its sex steroid binding protein. ${ }^{12}$ Both drugs have been reported to decrease self-reported deviant sexual drive, deviant fantasies and deviant sexual behaviour. ${ }^{12}$ However, there has been a lack of double-blind controlled trials, due to the ethical implications of an increased reoffending rate in the 'non-treatment' control group. Interestingly, no difference has been found in the pre-treatment serum testosterone levels between different categories of offenders, so that there is no theoretical reason to expect a specific benefit in a particular offender subgroup. $^{12}$

Kravitz et $a /^{13}$ reported that the onset of action of MPA in suppressing deviant sexual activities occurs within two weeks, with the suppression of non-deviant sexual behaviour being delayed for two to 10 weeks. Testosterone levels were found to return to baseline levels within 10 months after stopping treatment, this time-lag being somewhat longer in older men. Important side-effects related to this treatment include weight gain, gynaecomastia and an increased risk of heart disease. The side-effect profile of CPA and MPA have limited their usefulness as up to half of subjects default from treatment, compared to one third for cognitive-behavioural therapy. ${ }^{14}$

A more recent addition to the anti-androgenic armamentarium is triptorelin, a gonadotrophin releasing hormone analogue (GnRHA). In one small open label trial, intra-muscular administration of triptorelin lead to a cessation of deviant sexual behaviour in five of the six subjects studied, associated with a gradual decrease in the serum testosterone to castration levels. In one case, relapse occurred within 10 weeks of stopping treatment. The only side effects reported were those associated with hypo-androgenism, suggesting an advance on the previously available anti-androgenic drugs. A more recent study suggests that triptorelin has a good treatment effect in child molesters. ${ }^{15}$

The neurotransmitter serotonin appears to act centrally to decrease the release of dopamine in the midbrain and brainstem, which in turn appears to be associated with a decrease in sexual drive..$^{12}$ It is unclear whether this is a direct effect or a collateral effect of serotonin in relieving depression and anxiety and in decreasing impulsive behaviour. For this reason, drugs from the selective serotonin reuptake inhibitor (SSRI) group, which act to increase serotonergic function centrally, have been used in open label trials in the treatment of sexual deviance. ${ }^{16}$ While the results of these preliminary studies are encouraging, it is too early to draw definitive conclusions about the role of the SSRIs in the treatment of sex offenders). ${ }^{17}$

Sexual aggression is a complex phenomenon that has an intimate relationship with abnormal psychological functioning that amounts to a psychiatric disorder in a majority of sexual offenders. While psychotherapy addressing the cognitive distortions and social skills deficits that characterise many offenders is currently the preferred treatment option for managing sexual aggression per se, pharmacotherapy provides an alternative mode of treatment in some cases, and a combination of both psychological and drug treatments may improve treatment outcome. In any case, the very high prevalence of co-morbid psychiatric disorders requires the close involvement of the psychiatrist in any multidisciplinary approach to managing this complex patient group.

\section{References}

1. McGee H, Garavan R, deBarra M, Byrne J, Conroy R. The SAVI Report: Sexual Abuse and Violence in Ireland. Dublin; Liffey Press, 2002

2. McGrath R. Sex-offender risk assessment and disposition planning: a review of empirical findings. Int J Offender Therapy and Comparative Criminology, 1991; 35(4): 328-50.

3 . Gibbons $P$. The relationship of psychosocial characteristics of sex offenders and sexual aggression: A review. (2003a) In Press (Irish Journal of Psychological Medicine).

4. Kafka MP, Hennen J. A DSM-IV Axis I comorbidity study of males $(n=120)$ with paraphilias and paraphilia-related disorders. Sex Abuse, 2002; 14(4):349-66.

5. Raymond NC, Coleman E, Ohlerking F, Christenson GA, Miner M. Psychiatric comorbidity in pedophilic sex offenders. Am J Psychiatry, 2000; 157(5): 838.

6. Seto MC, Barbaree HE. The role of alcohol in sexual aggression. Clinical Psychology Review, 1995; 15(6): 545-66.

7. Beckett RC. Community treatment in the UK. In: Marshall W, Fernandez Y, Hudson S, Ward $T$ (Ed.s). Sourcebook of treatment programmes for sexual offenders. New York: Plenum Press, 1998

8. Salter AC. Treating child sex offenders: a practical guide. Newbury Park: Sage, 1988 9. Gibbons P. Recidivism and risk assessment in sex offenders:A Review. (2003b). In Press. Ir JPsych Med.

10. Melella JT, Travin S, Cullen K. Legal and ethical issues in the use of antiandrogens in treating sex offenders. Bulletin Am Acad Psychiatry and the Law, 1989;17(3): 223-32.

11. Murray JB. Psychopharmacological therapy of deviant sexual behavior. Journal of General Psychology, 1988; 115(1):101-10.

12. Haywood T, Cavanaugh JL. Sexual deviancy. Current Opinion in Psychiatry, 1996; 9(6): 384-8.

13. Kravitz HM, Haywood HW, Kelly JR, Wahlstrom C, Liles S, Cavanaugh JL. Medroxyprogesterone andparaphiles: do testosterone levels matter? Bulletin Am Acad Psychiatry and the Law, 1996; 24: 19-33.

14. Hall GCN. Sexual offender recidivism revisited: A meta-analysis of recent treatment studies. J Consulting Clinical Psychology, 1995; 63(5): 802-809.

15. Rosier A. Treatment of men with paraphilia with a long-acting analogue of gonadotrophinreleasing hormone. NEJM 1998; 338: 416-422.

16. Kafka MP. Sertraline pharmacotherapy for paraphilias and paraphilia related disorders: an open trial. Annals of Clinical Psychiatry, 1994; 6: 189-95.

17. Lehne G, Kate T, Berlin F. Treatment of sexual paraphilias: a review of the 1999-2000 literature. Current Opinion in Psychiatry 2000; 13(6): 569-573. 